Publ. Mat. (2007), 221-244

Proceedings of the Primeras Jornadas de Teoría de Números.

\title{
ON THE VALUE-DISTRIBUTION OF EPSTEIN ZETA-FUNCTIONS
}

\author{
JÖRN STEUding
}

\begin{abstract}
We investigate the value-distribution of Epstein zeta-functions $\zeta(s ; \mathcal{Q})$, where $\mathcal{Q}$ is a positive definite quadratic form in $n$ variables. We prove an asymptotic formula for the number of $c$-values, i.e., the roots of the equation $\zeta(s ; \mathcal{Q})=c$, where $c$ is any fixed complex number. Moreover, we show that, in general, these $c$-values are asymmetrically distributed with respect to the critical line $\operatorname{Re} s=\frac{n}{4}$. This complements previous results on the zero-distribution [30].
\end{abstract}

\section{Introduction and examples}

In the beginning of the twentieth century, Paul Epstein [12] introduced zeta-functions associated with quadratic forms. These so-called Epstein zeta-functions are interesting analytical objects which play a central role in algebraic number theory, the theory of modular forms (see Siegel's monograph [26]), and, recently, in chemistry and physics (see $[\mathbf{3}]$ and $[\mathbf{1 1}]$ ).

Let $\mathcal{Q}$ be a positive definite $n \times n$ matrix with integer entries and write $\mathcal{Q}[\mathbf{x}]=\mathbf{x}^{\mathrm{t}} \mathcal{Q} \mathbf{x}$ for the associated quadratic form. The Epstein zeta-function attached to $\mathcal{Q}$ is given by

$$
\zeta(s ; \mathcal{Q})=\sum_{0 \neq \mathbf{x} \in \mathbb{Z}^{n}} \mathcal{Q}[\mathbf{x}]^{-s} ;
$$

this Dirichlet series converges absolutely for $\operatorname{Re} s>\frac{n}{2}$, and uniformly in any compact subset. $\zeta(s ; \mathcal{Q})$ has an analytic continuation throughout the complex plane, except for a simple pole at $s=\frac{n}{2}$. The Epstein zeta-function satisfies a functional equation of the Riemann-type:

$$
\text { (1) } \quad \pi^{-s} \Gamma(s) \zeta(s ; \mathcal{Q})=(\operatorname{det} \mathcal{Q})^{-\frac{1}{2}} \pi^{s-\frac{n}{2}} \Gamma\left(\frac{n}{2}-s\right) \zeta\left(\frac{n}{2}-s ; \mathcal{Q}^{-1}\right) .
$$

2000 Mathematics Subject Classification. 11M41.

Key words. Epstein zeta-functions, quadratic forms, value-distribution, Nevanlinna theory. 
If $\mathcal{Q}$ is unimodular, i.e., if $\mathcal{Q} \in \mathrm{SL}_{n}(\mathbb{Z})$, then $\zeta(s ; \mathcal{Q})=\zeta\left(s ; \mathcal{Q}^{-1}\right)$, and the Epstein zeta-function is symmetric. In analogy to the case of the Riemann zeta-function, we call the vertical line $\operatorname{Re} s=\frac{n}{4}$, passing through the point of symmetry of the functional equation, the critical line (it should be noted that some authors call the lines $\operatorname{Re} s=\frac{1}{2}$ and $\operatorname{Re} s=\frac{n-1}{2}$ critical). Equation (1) implies the vanishing of $\zeta(s ; \mathcal{Q})$ at the so-called trivial zeros $s=-m, m \in \mathbb{N}$; all other zeros are said to be nontrivial and are denoted by $\rho=\beta+i \gamma$. As in the case of the Riemann zetafunction, very little is known about the distribution of the nontrivial zeros of Epstein zeta-functions.

We start with some examples. Denote by $\mathbf{1}_{n}$ the $n$-dimensional unit matrix. Then

$$
\zeta\left(s ; \mathbf{1}_{1}\right)=2 \zeta(2 s) \quad \text { and } \quad \zeta\left(s ; \mathbf{1}_{2}\right)=4 \zeta(s) L(s ; \chi),
$$

where $\zeta(s)$ is the Riemann zeta-function and $L(s ; \chi)$ is the Dirichlet $L$-function to the non-principal character $\chi \bmod 4$; it should be noted that $\zeta\left(s ; \mathbf{1}_{2}\right)$ is the Dedekind zeta-function of the Gaussian field $\mathbb{Q}(i)$. Moreover,

$$
\begin{aligned}
& \zeta\left(s ; \mathbf{1}_{4}\right)=8\left(1-2^{2-s}\right) \zeta(s) \zeta(s-1), \\
& \zeta\left(s ; \mathcal{S}_{8}\right)=240 \cdot 2^{-s} \zeta(s) \zeta(s-3),
\end{aligned}
$$

where $\mathcal{S}_{8}$ is the matrix

$$
\mathcal{S}_{8}=\left(\begin{array}{cc}
2 \cdot \mathbf{1}_{4} & \mathcal{A} \\
-\mathcal{A} & 2 \cdot \mathbf{1}_{4}
\end{array}\right) \quad \text { with } \quad \mathcal{A}:=\left(\begin{array}{cccc}
0 & 1 & 1 & 1 \\
-1 & 0 & -1 & 1 \\
-1 & 1 & 0 & -1 \\
-1 & -1 & 1 & 0
\end{array}\right)
$$

A completely different example is given by

$$
\zeta\left(s ; \mathcal{L}_{24}\right)=\frac{65520}{691}\{\zeta(s) \zeta(s-11)-L(s ; \Delta)\},
$$

where $L(s ; \Delta):=\sum_{m=1}^{\infty} \tau(m) m^{-s}$ is the $L$-function attached to Ramanujan's $\tau$-function, given by

$$
\Delta(q)=\sum_{m=1}^{\infty} \tau(m) q^{m}=q \prod_{m=1}\left(1-q^{m}\right)^{24}
$$

with $q:=\exp (2 \pi i \tau)$ and $\tau$ from the upper half-plane, and $\mathcal{L}_{24}$ is the matrix related to the Leech lattice defined by

$$
\mathcal{L}_{24}=\left(\begin{array}{cc}
4 \cdot \mathbf{1}_{12} & \mathcal{B}-2 \cdot \mathbf{1}_{12} \\
\mathcal{B}^{\mathrm{t}}-2 \cdot \mathbf{1}_{12} & 4 \cdot \mathbf{1}_{12}
\end{array}\right), \quad \text { where } \quad \mathcal{B}:=\left(\begin{array}{cc}
0 & \mathbf{e}^{\mathbf{t}} \\
-\mathbf{e} & \mathcal{C}
\end{array}\right)
$$


$\mathbf{e}:=(1, \ldots, 1)^{\mathrm{t}}, \mathcal{C}:=\left(\left(\frac{k-\ell}{11}\right)\right)_{1 \leq k, \ell<11}$, and $\left(\frac{\dot{11}}{11}\right)$ is the Legendre symbol mod11 (this construction of the Leech lattice is due to McKay (cf. [6], resp. [19]). Assuming the truth of the Riemann hypothesis for $\zeta(s)$ and $L(s, \chi)$ (i.e., all nontrivial zeros lie on the line $\operatorname{Re} s=\frac{1}{2}$ ), all nontrivial zeros of the functions in (2) lie on the critical lines $\operatorname{Re} s=\frac{1}{4}$ and $\operatorname{Re} s=\frac{1}{2}$, respectively; unconditionally, we know that a positive proportion of the nontrivial zeros of $\zeta(s ; \mathcal{Q})$ lie on these critical lines which follows from the same fact for the Riemann zeta-function $\zeta(s)$ (see [31]) and Formula (6) below. However, the distribution of zeros of the other examples is rather different. Example (4) is expected to have all its nontrivial zeros on the lines $\operatorname{Re} s=\frac{1}{2}$ and $\operatorname{Re} s=\frac{7}{2}$ while Example (3) is expected to have most of its zeros on the lines $\operatorname{Re} s=\frac{1}{2}$ and $\operatorname{Re} s=\frac{3}{2}$ (again by the Riemann hypothesis), but, in the latter example, infinitely many lie on $\operatorname{Re} s=2$. In both examples there are no zeros on the respective critical lines since all nontrivial zeros of $\zeta(s)$ lie inside the critical strip $0<\operatorname{Re} s<1$ (see again [31]). Nothing definite is known about the distribution of zeros of the function (5).

Epstein zeta-functions associated with binary quadratic forms are somehow special. For $n=2$ the functions $\zeta(s ; \mathcal{Q})$ and $\zeta\left(s ; \mathcal{Q}^{-1}\right)$ are equal up to a constant factor, which gives the functional equation (1) a pleasant form. The zero-distribution of these Epstein zeta-functions was first studied by Potter and Titchmarsh [24]. They proved that infinitely many zeros lie on the critical line $\operatorname{Re} s=\frac{1}{2}$. Bateman and Grosswald [1] showed that Epstein zeta-functions attached to positive definite quadratic forms $a x^{2}+b x y+c y^{2}$ with discriminant $\Delta:=b^{2}-4 a c$ have a real zero between $\frac{1}{2}$ and 1 if

$$
k:=\frac{\sqrt{|\Delta|}}{2 a}>7.00556
$$

in fact, this result was announced by Chowla and Selberg [5] but they never published a proof. Deuring $[\mathbf{8}]$ and Stark $[\mathbf{2 7}]$ showed that all zeros of these Epstein zeta-functions in the rectangle $-1<\operatorname{Re} s<2$, $|\operatorname{Im} s| \leq 2 k$ lie on the critical line $\operatorname{Re} s=\frac{1}{2}$ and are simple with the exception of two real zeros between 0 and 1 , provided $k$ is sufficiently large. The Epstein zeta-function associated with binary quadratic forms either have an Euler product representation or are a linear combination of Hecke $L$-functions for ideal class characters, depending on the class number of the underlying quadratic form being equal to or larger than 1 . Hejhal [15] and Bombieri and Hejhal [2] proved for the latter Epstein zeta-functions that almost all zeros lie on the critical line subject to the truth of the Generalized Riemann hypothesis - more precisely, the 
Riemann hypothesis for Hecke $L$-functions - in combination with an unproved but widely believed conjecture on the spacing of consecutive zeros of these Hecke $L$-functions. Davenport and Heilbronn $[\mathbf{7}]$ proved that $\zeta(s ; \mathcal{Q})$ has an infinitude of zeros in the half-plane of absolute convergence $\operatorname{Re} s>1$ if the class number is greater than 1. For Epstein zeta-functions attached to certain quadratic forms of rank $n=4$, Fujii [14] obtained similar results as for binary quadratic forms.

\section{Statement of the main results}

This paper can be regarded as sequel to [30]. In this paper we have shown that the mean-value of the real parts of the nontrivial zeros of the Epstein zeta-function is equal to the point of symmetry of the functional equation, that is the abscissa of the critical line $\operatorname{Re} s=\frac{n}{4}$. Nevertheless, we have also shown that a generic Epstein zeta-function associated with quadratic forms in more than two variables has an asymmetric zerodistribution. Before we can put these results in a precise form, we have to introduce some further notation. Let $N(T ; \mathcal{Q})$ count the number of nontrivial zeros $\rho=\beta+i \gamma$ of $\zeta(s ; \mathcal{Q})$ with $|\gamma| \leq T$. Denote by $\mathrm{m}_{j}(\mathcal{Q})$ the $j$ th minimum of the values of the quadratic form $\mathcal{Q}[\mathbf{x}]$ for $0 \neq \mathbf{x} \in \mathbb{Z}^{n}$, i.e.,

$$
\begin{aligned}
\mathrm{m}_{1}(\mathcal{Q}) & =\min \{m \in \mathbb{N} \mid \exists \mathbf{x}: \mathcal{Q}[\mathbf{x}]=m\}, \\
\mathrm{m}_{j+1}(\mathcal{Q}) & =\min \left\{m \in \mathbb{N} \mid m>\mathrm{m}_{j}(\mathcal{Q}), \exists \mathbf{x}: \mathcal{Q}[\mathbf{x}]=m\right\} \quad \text { for } \quad j \in \mathbb{N} .
\end{aligned}
$$

Finally, let $\mathrm{N}(\mathcal{Q})$ count the number of $\mathbf{x} \in \mathbb{Z}^{n}$ for which $\mathcal{Q}[\mathbf{x}]=\mathrm{m}_{1}(\mathcal{Q})$ and, for $m \in \mathbb{N}$, define $r(m ; \mathcal{Q})=\sharp\left\{\mathbf{x} \in \mathbb{Z}^{n}: \mathcal{Q}[\mathbf{x}]=m\right\}$. Then

Theorem 0. As $T \rightarrow \infty$,

$$
N(T ; \mathcal{Q})=\frac{2 T}{\pi} \log \frac{T}{\pi e \sqrt{\mathrm{m}_{1}(\mathcal{Q}) \mathrm{m}_{1}\left(\mathcal{Q}^{-1}\right)}}+O(\log T),
$$

and

(7) $\sum_{|\gamma| \leq T}\left(\beta-\frac{n}{4}\right)=\frac{T}{\pi} \log \left((\operatorname{det} \mathcal{Q})^{-\frac{1}{2}} \frac{\mathrm{N}\left(\mathcal{Q}^{-1}\right)}{\mathrm{N}(\mathcal{Q})}\left(\frac{\mathrm{m}_{1}(\mathcal{Q})}{\mathrm{m}_{1}\left(\mathcal{Q}^{-1}\right)}\right)^{\frac{n}{4}}\right)+O(\log T)$.

Equation (6) is a Riemann-von Mangoldt formula for Epstein zetafunctions (and it appears in slightly different form in the papers of Bombieri and Hejhal [2], Stark [27], and others). The asymptotic formula (7) measures asymmetries in the zero-distribution with respect to 
the critical line. If the quantity

$$
\Sigma(\mathcal{Q}):=\log \left((\operatorname{det} \mathcal{Q})^{-\frac{1}{2}} \frac{\mathrm{N}\left(\mathcal{Q}^{-1}\right)}{\mathrm{N}(\mathcal{Q})}\left(\frac{\mathrm{m}_{1}(\mathcal{Q})}{\mathrm{m}_{1}\left(\mathcal{Q}^{-1}\right)}\right)^{\frac{n}{4}}\right)
$$

is not equal to 0 , then $\zeta(s ; \mathcal{Q})$ has infinitely many zeros off the critical line $\operatorname{Re} s=\frac{n}{4}$. The Examples $(2)-(5)$ all have $\Sigma(\mathcal{Q}) \equiv 0$; however, the generic example has an asymmetric distribution of nontrivial zeros. If compared with the total number of zeros, the possible asymmetries are small. The combination of both asymptotic formulae of the theorem above leads to

$$
\frac{1}{N(T ; \mathcal{Q})} \sum_{|\gamma| \leq T} \beta=\frac{n}{4}+O\left(\frac{1}{\log T}\right),
$$

and thus the mean-value of the real parts of the nontrivial zeros of $\zeta(s ; Q)$ exists and is equal to $\frac{n}{4}$. In this paper we are concerned with the valuedistribution of Epstein zeta-functions in general, not necessarily with the distribution of the zeros.

Let $c$ be any complex number. Levinson [21] proved that all but $O\left(\frac{T \log T}{\log \log T}\right)$ of the roots of $\zeta(s)=c$ in $T<\operatorname{Im} s<2 T$ lie in

$$
\left|\operatorname{Re} s-\frac{1}{2}\right|<\frac{(\log \log T)^{2}}{\log T} .
$$

Thus, the $c$-values of the zeta-function are clustered around the critical line $\operatorname{Re} s=\frac{1}{2}$. In particular, it follows from Levinson's result that classic estimates for the number $N(\sigma, T)$ of zeros of $\zeta(s)$ in the region $\operatorname{Re} s>\alpha$ and $0<\operatorname{Im} s \leq T$ of the form $N(\sigma, T)=O(T)$ for fixed $\sigma>\frac{1}{2}$ do not indicate the truth of the Riemann hypothesis (as it is written in $[\mathbf{1 0}]$ ); nevertheless, such density theorems (and their refinements) play a central role in the theory of zeta-functions. On behalf of (2), the Riemann zetafunction is a special example of an Epstein zeta-function. It is natural to ask whether the clustering of $c$-values is also true for Epstein zetafunctions.

Let $c$ be a fixed complex number. The $c$-values of $\zeta(s ; \mathcal{Q})$ are the roots of the equation

$$
\zeta(s ; \mathcal{Q})=c,
$$

which we denote by $\rho_{c}=\beta_{c}+i \gamma_{c}$. We shall show that the distribution of the $c$-values is quite similar to the distribution of zeros as indicated by Theorem 0 . For this purpose let $N_{c}(\sigma, T ; \mathcal{Q})$ count the number of $c$-values of $\zeta(s ; \mathcal{Q})$ with $\beta_{c}>\sigma$ and $0<\gamma_{c} \leq T$. Here we have to add the condition on the real part to be bounded below in order to exclude 
an infinity of $c$-values lying close to the negative real axis (this will be explained in Section 4). Our first theorem gives an asymptotic formula for the counting function $N_{c}(\sigma, T ; \mathcal{Q})$ of the $c$-values as well as one for sums measuring asymmetries in their distribution with respect to the critical line.

Theorem 1. Let $c$ be any complex number. Then, for any $\sigma<0$, as $T \rightarrow \infty$,

$$
N_{c}(\sigma, T ; \mathcal{Q})=\frac{T}{\pi} \log \frac{T}{\pi e \sqrt{\mu(c, \mathcal{Q}) \mathrm{m}_{1}\left(\mathcal{Q}^{-1}\right)}}+O(\log T),
$$

(9) $\sum_{T<\gamma_{c} \leq 2 T}\left(\beta_{c}-\frac{n}{4}\right)=\frac{T}{\pi} \log \frac{\nu(c, \mathcal{Q}) \mathrm{N}\left(\mathcal{Q}^{-1}\right)}{\sqrt{\operatorname{det} \mathcal{Q}} \mathrm{m}_{1}\left(\mathcal{Q}^{-1}\right)^{\frac{n}{4}}}+O(\log T)$,

where

$$
\mu(c, \mathcal{Q}):= \begin{cases}1 & \text { if } c \notin\{0, \mathrm{~N}(\mathcal{Q})\}, \mathrm{m}_{1}(\mathcal{Q})=1 \\ & \text { or } c \neq 0, \mathrm{~m}_{1}(\mathcal{Q})>1 \\ \mathrm{~m}_{1}(\mathcal{Q}) & \text { if } c=0 \\ \mathrm{~m}_{2}(\mathcal{Q}) & \text { if } c=\mathrm{N}(\mathcal{Q}), \mathrm{m}_{1}(\mathcal{Q})=1\end{cases}
$$

and

$$
\nu(c, \mathcal{Q}):= \begin{cases}\frac{1}{|\mathrm{~N}(\mathcal{Q})-c|} & \text { if } c \notin\{0, \mathrm{~N}(\mathcal{Q})\}, \mathrm{m}_{1}(\mathcal{Q})=1, \\ \frac{1}{|c|} & \text { if } c \neq 0, \mathrm{~m}_{1}(\mathcal{Q})>1 \\ \frac{\mathrm{m}_{1}(\mathcal{Q})^{\frac{n}{4}}}{\mathrm{~N}(\mathcal{Q})} & \text { if } c=0 \\ \frac{\mathrm{m}_{2}(\mathcal{Q})^{\frac{n}{4}}}{r\left(\mathrm{~m}_{2}(\mathcal{Q})\right)} & \text { if } c=\mathrm{N}(\mathcal{Q}), \mathrm{m}_{1}(\mathcal{Q})=1 .\end{cases}
$$

It is interesting to see that, in general, the $c$-values are asymmetrically distributed with respect to the critical line. However, there exist some cases with $\sum\left(\beta_{c}-\frac{n}{4}\right) \equiv 0$; e.g., if $\mathrm{m}_{1}(\mathcal{Q})=1$, then all $c \notin\{0, \mathrm{~N}(\mathcal{Q})\}$ lying on the circle

$$
|c-\mathrm{N}(\mathcal{Q})|=\frac{\mathrm{N}\left(\mathcal{Q}^{-1}\right)}{\sqrt{\operatorname{det} \mathcal{Q}} \mathrm{m}_{1}\left(\mathcal{Q}^{-1}\right)^{\frac{n}{4}}} .
$$

We can also recover from this theorem part of the results on the distribution of $c$-values of Euler products obtained in [28], [29], for example, 
by (2) those for $\zeta(s)$ and the Dedekind zeta-function of $\mathbb{Q}(i)$. Moreover, we may deduce that the number of 1 -values $\rho_{1}=\beta_{1}+i \gamma_{1}$ of $\zeta(s)$ with $\beta_{1}>0$ and $0<\gamma_{1} \leq T$ is equal to

$$
\frac{T}{2 \pi} \log \frac{T}{2 \pi e}+O(\log T)
$$

and that they are symmetrically distributed with respect to the critical line; this case was not considered in $[\mathbf{2 8}],[\mathbf{2 9}]$. Note that the valuedistribution in the lower half-plane is reflected by

$$
\zeta(\bar{s} ; \mathcal{Q})=\overline{\zeta(s ; \mathcal{Q})} \text {. }
$$

Besides Theorem 1, we shall obtain information about the distribution of $c$-values to the left and to the right of the critical line. Define the counting functions (according multiplicities)

$$
\begin{aligned}
& \mathcal{N}_{c}^{+}(\sigma, T ; \mathcal{Q})=\sharp\left\{\rho_{c}: T<\gamma_{c} \leq 2 T, \beta_{c}>\sigma\right\}, \\
& \mathcal{N}_{c}^{-}(\sigma, T ; \mathcal{Q})=\sharp\left\{\rho_{c}: T<\gamma_{c} \leq 2 T, \beta_{c} \leq \sigma\right\} .
\end{aligned}
$$

Further let $\mathcal{N}_{c}(T, \mathcal{Q})$ count the number of all $c$-values with $T<\gamma_{c} \leq 2 T$, i.e.,

$$
\mathcal{N}_{c}(T ; \mathcal{Q})=\mathcal{N}_{c}^{+}(\sigma, T ; \mathcal{Q})+\mathcal{N}_{c}^{-}(\sigma, T ; \mathcal{Q})
$$

Here we do not have to add a lower bound for the real parts since there do not exist $c$-values with $\beta_{c}<0$ and $\gamma_{c} \geq T$ as $T \rightarrow \infty$.

Theorem 2. Let $c$ be any complex number and $b>\max \left\{\frac{1}{4}, \frac{n-1}{2}\right\}$ fixed. Then, as $T \rightarrow \infty$,

$$
\sum_{\substack{\beta_{c}>b \\ T<\gamma_{c} \leq 2 T}}\left(\beta_{c}-b\right) \ll T .
$$

Moreover, for fixed $0<\sigma<\frac{n}{4}$ and any $\epsilon>0$,

$$
\mathcal{N}_{c}^{+}\left(\max \left\{\frac{1}{4}, \frac{n-1}{2}\right\}+\epsilon, T ; \mathcal{Q}\right) \ll T,
$$

and

$$
\mathcal{N}_{c}^{-}(\sigma, T ; \mathcal{Q}) \leq \begin{cases}\frac{n-2+\epsilon}{2 n-2-4 \sigma} \mathcal{N}_{c}(T ; \mathcal{Q}) & \text { if } n \geq 2, \\ O(T) & \text { if } n=1 .\end{cases}
$$

First, we shall discuss the quality of the estimate (14). Voronin [32] (see also [18]) has shown that Epstein zeta-functions of rank 2 and class number greater than 1 have $\gg T$ many zeros in any rectangle $\operatorname{Re} s>\alpha$, $0<\operatorname{Im} s \leq T$, where $\alpha \in\left(\frac{1}{2}, 1\right)$ is fixed, as $T \rightarrow \infty$; his proof relies on the universality of these Epstein zeta-functions. This complements the result of Davenport and Heilbronn mentioned in the previous section. 
Furthermore, it shows that the bound is sharp for rank $n=2$. Another example of this type of rank $n=4$ is given by (3). Also the range for $b$ is sharp as it follows from the latter example and from the results of Bombieri and Hejhal [2], [15] for rank $n=2$.

We are neither able to prove a generalization of Levinson's aforementioned result on the clustering of $c$-values of $\zeta(s)$ nor to generalize the results of Bombieri and Hejhal on the localization of almost all nontrivial zeros on the critical line. Equation (15) with $\sigma=\frac{n}{2}-\max \left\{\frac{1}{4}, \frac{n-1}{2}\right\}-\epsilon$ implies

$$
\mathcal{N}_{c}^{-}\left(\frac{n}{2}-\max \left\{\frac{1}{4}, \frac{n-1}{2}\right\}-\epsilon, T ; \mathcal{Q}\right) \leq \frac{1}{2} \mathcal{N}_{c}(T ; \mathcal{Q})
$$

In conjunction with (14) we obtain

$$
\begin{aligned}
\sharp\left\{\rho_{c}=\right. & \beta_{c}+i \gamma_{c} \mid \frac{n}{2}-\max \left\{\frac{1}{4}, \frac{n-1}{2}\right\} \\
& \left.-\epsilon<\beta_{c} \leq \max \left\{\frac{1}{4}, \frac{n-1}{2}\right\}+\epsilon, T \leq \gamma_{c} \leq 2 T\right\} \geq \frac{1}{2} \mathcal{N}_{c}(T ; \mathcal{Q}) .
\end{aligned}
$$

Besides, we get clustering around the critical line $\operatorname{Re} s=\frac{n}{4}$ for $n=1,2$; however, this cannot hold for $n>2$ in general as it follows from the examples in (3) and (4). We conjecture that almost all $c$-values (including zeros) lie in or close to the strip $\frac{1}{2} \leq \operatorname{Re} s \leq \frac{n-1}{2}$ if $n>2$. We conclude this discussion with another interesting, related result in this direction: Siegel [25] proved for $n \geq 12$ and $\mathcal{Q}$ belonging to the genus of $\mathbf{1}_{n}$ that the number of zeros in $2 \leq \operatorname{Re} s \leq \frac{n}{2}-2,0<\operatorname{Im} s \leq T$ is $\frac{T}{\pi} \log 2+O(1)$; furthermore, almost all zeros do not lie in the neighborhood of the critical line $\operatorname{Re} s=\frac{n}{4}$. In conjunction with (14) we can sharpen Siegel's estimate for this special example slightly: almost all zeros of $\zeta\left(s ; \mathbf{1}_{n}\right)$ with $n \geq 12$ lie in the strips $0 \leq \operatorname{Re} s<2$ and $\frac{n}{2}-2<\operatorname{Re} s \leq \frac{n-1}{2}+\epsilon$ for any $\epsilon>0$.

The results can be generalized to rational quadratic forms simply by multiplication with the least common multiple of the denominators of all coefficients. However, it seems to be difficult to study quadratic forms which are not proportional to a rational form by the same methods.

\section{The mean-square and other preliminaries}

We start with some growth estimates. We can rewrite the functional equation (1) as

$$
\zeta(s ; \mathcal{Q})=\Xi(s ; \mathcal{Q}) \zeta\left(\frac{n}{2}-s ; \mathcal{Q}^{-1}\right)
$$

where

$$
\Xi(s ; \mathcal{Q}):=(\operatorname{det} \mathcal{Q})^{-\frac{1}{2}} \pi^{2 s-\frac{n}{2}} \frac{\Gamma\left(\frac{n}{2}-s\right)}{\Gamma(s)} .
$$


In the sequel we shall write the complex variable, as it is tradition, as $s=\sigma+i t$ with $\sigma, t \in \mathbb{R}$ and $\sqrt{-1}$. By Stirling's formula,

$$
|\Xi(\sigma+i t ; \mathcal{Q})|=(\operatorname{det} \mathcal{Q})^{-\frac{1}{2}}\left(\frac{\pi}{|t|}\right)^{2 \sigma-\frac{n}{2}}\left(1+O\left(|t|^{-1}\right)\right)
$$

for $|t| \geq 1$ and bounded $\sigma$. Hence, an application of the PhragménLindelöf principle yields

$$
\zeta(\sigma+i t ; \mathcal{Q}) \ll|t|^{\max \left\{\frac{n}{2}-\sigma, 0\right\}+\epsilon}
$$

as $|t| \rightarrow \infty$, where the implicit constant depends only on $\mathcal{Q}$; here and in the sequel $\epsilon$ denotes an arbitrary small positive constant, not necessarily the same at each appearance. This estimate shows that $\zeta(s ; \mathcal{Q})$ is a function of finite order.

However, we can show a bit more. Chandrasekharan and Narasimhan [4] obtained an approximate functional equation for a quite general class of zeta-functions. It is not difficult to deduce from their general result:

Lemma 3. We have

$$
\zeta(s ; \mathcal{Q})=\sum_{\substack{0 \neq \mathbf{x} \in \mathbb{Z} \\ \mathcal{Q}[\mathbf{x}] \leq t / \pi}} \mathcal{Q}[\mathbf{x}]^{-s}+\Xi(s ; \mathcal{Q}) \sum_{\substack{0 \neq \mathbf{x} \in \mathbb{Z} \\ \mathcal{Q}[\mathbf{x}] \leq t / \pi}} \mathcal{Q}^{-1}[\mathbf{x}]^{s-\frac{n}{2}}+O\left(t^{\frac{n}{2}-\sigma-1} \log t\right)
$$

uniformly in $s$ for $\frac{n}{4}-\frac{1}{2}\left[\frac{n+1}{2}\right] \leq \sigma \leq B$ and $t \geq 2$, where $B$ is any fixed positive constant and $[x]$ denotes, as usual, the largest integer less than or equal to $x$.

Landau's Tauberian theorem (see [20]) yields

$$
\sum_{\substack{\mathbf{0} \neq \mathbf{x} \in \mathbb{Z} \\ \mathcal{Q}[\mathbf{x}] \leq X}} \mathcal{Q}[\mathbf{x}]^{1-\frac{n}{2}} \sim \frac{\pi^{\frac{n}{2}}}{\Gamma\left(\frac{n}{2}\right) \sqrt{\operatorname{det} \mathcal{Q}}} X,
$$

where the constant factor on the right hand side is the residue of $\zeta(s ; \mathcal{Q})$ at $s=\frac{n}{2}$. This together with Lemma 3 and (18) lead to the estimate

$$
\zeta\left(\frac{n}{4}+i t\right) \ll t^{\frac{n}{4}}
$$

as $t \rightarrow \infty$, which is a slight improvement upon (19) on the critical line $\operatorname{Re} s=\frac{n}{4}$. For certain classes of quadratic forms Fomenko [13] obtained sharper bounds.

Following the lines of Chandrasekharan and Narasimhan [4] one can deduce from Lemma 3 
Lemma 4. Let $n \geq 2$. For fixed $\sigma>\frac{n-1}{2}$, as $T \rightarrow \infty$,

$$
\int_{1}^{T}|\zeta(\sigma+i t ; \mathcal{Q})|^{2} \mathrm{~d} t=T \sum_{m=1}^{\infty} \frac{r(m)^{2}}{m^{2 \sigma}}+O\left(T^{\max \left\{\frac{n+1}{2}-\sigma, 0\right\}+\epsilon}\right),
$$

where $r(m):=r(m ; \mathcal{Q})$.

We leave out the proof since this mean-square estimate follows immediately from a far more general result due to Kanemitsu et al. [17]. To see this note for the number of representations of an integer $m$ by a quadratic form $\mathcal{Q}[\mathbf{x}]$ the estimate

$$
r(m ; \mathcal{Q}) \ll m^{\frac{n}{2}-1}
$$

as $m \rightarrow \infty$ (see [9] and [16]); the latter estimate is stronger than (20).

Example (4) shows that the range for $\sigma$ in Lemma 4 cannot be extended since

$$
\int_{1}^{T}\left|\zeta\left(\frac{7}{2}+i t ; \mathcal{S}_{8}\right)\right|^{2} \mathrm{~d} t \gg \int_{1}^{T}\left|\zeta\left(\frac{1}{2}+i t\right)\right|^{2} \mathrm{~d} t \asymp T \log T
$$

by a classic result of Hardy and Littlewood (see, for example, [18]) and the rough estimate $|\zeta(\sigma+i t)|>\exp \left(-\frac{2}{3} \zeta(\sigma)\right)$, valid for $\sigma>1$.

For the excluded case $n=1$, we note the classic mean-square formula

$$
\int_{1}^{T}|\zeta(\sigma+i t)|^{2} \mathrm{~d} t=\zeta(2 \sigma) T+O\left(T^{\max \left\{\frac{n+1}{2}-\sigma, 0\right\}+\epsilon}\right),
$$

valid for $\sigma>\frac{1}{2}$ (see [31]). This implies for (2) an asymptotic formula of this type for the range $\sigma>\frac{1}{4}$; namely,

$$
\int_{1}^{T}\left|\zeta\left(\sigma+i t ; \mathbf{1}_{1}\right)\right|^{2} \mathrm{~d} t=4 \zeta(4 \sigma) T+O\left(T^{\max \{1-\sigma, 0\}+\epsilon}\right) .
$$

The proof of Theorem 1 relies on a method of Levinson [21] and, in particular, an application of Littlewood's lemma which relates the zeros of an analytic function $f(s)$ with a contour integral over $\log f(s)$.

Lemma 5 (Littlewood). Let $a>b$ and let $f(s)$ be analytic on $\mathcal{R}:=$ $\{s \in \mathbb{C}: b \leq \operatorname{Re} s \leq a,|\operatorname{Im} s| \leq T\}$. Suppose that $f(s)$ does not vanish on the right edge $\sigma=a$ of $\mathcal{R}$. Let $\mathcal{R}^{\prime}$ be $\mathcal{R}$ minus the union of the horizontal cuts from the zeros of $f$ in $\mathcal{R}$ to the left edge of $\mathcal{R}$, and choose a single-valued branch of $\log f(s)$ in the interior of $\mathcal{R}^{\prime}$. Denote by $\nu(\sigma, T)$ the number of zeros $\rho=\beta+i \gamma$ of $f(s)$ inside the rectangle with $\beta>\sigma$ including zeros with $\gamma=T$ but not those with $\gamma=-T$. Then

$$
\int_{\partial \mathcal{R}} \log f(s) \mathrm{d} s=-2 \pi i \int_{b}^{a} \nu(\sigma, T) \mathrm{d} \sigma .
$$


We give a sketch of the simple proof. Cauchy's theorem implies $\int_{\partial \mathcal{R}^{\prime}} \log f(s) \mathrm{d} s=0$, and so the left-hand side of the formula of the lemma, $\int_{\partial \mathcal{R}}$, is minus the sum of the integrals around the paths hugging the cuts. Since the function $\log f(s)$ jumps by $2 \pi i$ across each cut (assuming for simplicity that the zeros of $f$ in $\mathcal{R}$ are simple and have different height; the general case is no harder), the quantity $\int_{\partial \mathcal{R}}$ is $-2 \pi i$ times the total length of the cuts, which is the right-hand side of the formula in the lemma. For more details we refer to Titchmarsh [31, $\S 9.9]$, or Littlewood's original paper [22].

\section{Application of Levinson's method}

Levinson introduced his method in order to prove his result on the $c$-values of $\zeta(s)$, mentioned in Section 2. The case of Epstein zeta-functions is by far more technical than the one of Riemann's zeta-function. For our purpose, we define, for $c \neq 0$,

$$
\begin{array}{ll}
\ell_{1}(s, \mathcal{Q})=\frac{\zeta(s ; \mathcal{Q})-c}{\mathrm{~N}(\mathcal{Q})-c} & \text { for } \quad c \neq \mathrm{N}(\mathcal{Q}), \\
\ell_{2}(s, \mathcal{Q})=\frac{\mathrm{m}_{2}(\mathcal{Q})^{s}}{r\left(\mathrm{~m}_{2}(\mathcal{Q})\right)}\{\zeta(s ; \mathcal{Q})-c\} & \text { for } \quad c=\mathrm{N}(\mathcal{Q})
\end{array}
$$

if $\mathrm{m}(\mathcal{Q})=1$, and

$$
\ell_{3}(s, \mathcal{Q})=\frac{1}{c}\{c-\zeta(s ; \mathcal{Q})\}
$$

if $\mathrm{m}(\mathcal{Q})>1$. Moreover, for $c=0$, let

$$
\ell_{4}(s, \mathcal{Q})=\frac{\mathrm{m}_{1}(\mathcal{Q})^{s}}{\mathrm{~N}(\mathcal{Q})} \zeta(s ; \mathcal{Q})
$$

Note that all functions $\ell_{j}(s ; \mathcal{Q})$ have a Dirichlet series expansion for $\sigma>\frac{n}{2}$, and satisfy the estimate

$$
\ell_{j}(s, \mathcal{Q})=1+\lambda^{-s}+O\left(\Lambda^{-\sigma}\right)
$$

as $\sigma \rightarrow \infty$, where $\lambda, \Lambda$ are constants satisfying $1<\lambda<\Lambda$, depending only on $\mathcal{Q}$ and $c$; to see this note that $\mathrm{N}(\mathcal{Q})=r\left(\mathrm{~m}_{1}(\mathcal{Q})\right)$ and

$$
\zeta(s ; \mathcal{Q})=\frac{\mathrm{N}(\mathcal{Q})}{\mathrm{m}_{1}(\mathcal{Q})^{s}}+\frac{r\left(\mathrm{~m}_{2}(\mathcal{Q})\right)}{\mathrm{m}_{2}(\mathcal{Q})^{s}}+\text { higher terms }
$$


Further, notice that the zeros of any $\ell_{j}(s, \mathcal{Q})$ correspond exactly to the $c$-values of $\zeta(s ; \mathcal{Q})$ (counting multiplicities). In order to treat all cases simultaneously, let

$$
\ell(s, \mathcal{Q})=\alpha \beta^{s}\{\zeta(s ; \mathcal{Q})-c\}
$$

stand for any of the functions $\ell_{j}(s, \mathcal{Q})$ with real constants $\alpha=\alpha(c, \mathcal{Q})$, $\beta=\beta(c, \mathcal{Q})$, corresponding to the above cases.

In the sequel we suppose that $n \geq 2$; the case of $n=1$ is covered by Levinson's results mentioned in Section 2; however, it can be treated similarly (the main difference is that the quadratic mean asymptotics from Lemma 4 have to be replaced by (21)).

We start with the proof of (13) of Theorem 2. In view of (22) there exists a positive real number $A$ depending on $c$ such that all real parts of the roots of $\ell(s ; \mathcal{Q})$ and of $\ell\left(s ; \mathcal{Q}^{-1}\right)$ (i.e., the real parts of the $c$-values of $\zeta(s ; \mathcal{Q})$ and of $\left.\zeta\left(s ; \mathcal{Q}^{-1}\right)\right)$ satisfy $\beta_{c}<A$; such a zero-free region exists since, by $(22), \ell(s ; \mathcal{Q})$ tends to 1 as $\sigma \rightarrow \infty$. We apply Littlewood's Lemma 5 to the function $f(s):=\left(s-\frac{n}{2}\right) \ell(s ; \mathcal{Q})$; note that $f(s)$ is an entire function (since we have removed the simple pole of $\zeta(s ; \mathcal{Q})$ at $s=\frac{n}{2}$ by multiplying with $\left.s-\frac{n}{2}\right)$ and that its zeros exactly correspond to the zeros of $\ell(s ; \mathcal{Q})$, resp. the $c$-values of $\zeta(s ; \mathcal{Q})$. Further, let $a$ be a parameter with $a>\max \{A+1, b\}$. Then Littlewood's Lemma 5, applied to the rectangle $\mathcal{R}$ with vertices $a+i T, a+2 i T, b+i T, b+2 i T$, gives

$$
\int_{\partial \mathcal{R}} \log \left(\left(s-\frac{n}{2}\right) \ell(s ; \mathcal{Q})\right) \mathrm{d} s=-2 \pi i \int_{b}^{a} \nu(\sigma, T) \mathrm{d} \sigma .
$$

First, we shall remove the factor $s-\frac{n}{2}$. Applying Littlewood's lemma once again, we get

$$
\int_{\partial \mathcal{R}} \log \left(s-\frac{n}{2}\right) \mathrm{d} s=-2 \pi i\left(\frac{n}{2}+b\right)
$$

and so the contribution of the factor $s-\frac{n}{2}$ in (24) is bounded. Hence, $(24)$ holds for $\ell(s ; \mathcal{Q})$ in place of $f(s)$ after adding an error term $O(1)$. Since

$$
\int_{b}^{a} \nu(\sigma, T) \mathrm{d} \sigma=\sum_{\substack{\beta_{c}>b \\ T<\gamma \leq 2 T}} \int_{b}^{\beta_{c}} \mathrm{~d} \sigma=\sum_{\substack{\beta_{c}>b \\ T<\gamma_{c} \leq 2 T}}\left(\beta_{c}-b\right),
$$


and this quantity is a real number, we get

$$
\begin{aligned}
2 \pi \sum_{\substack{\beta_{c}>b \\
T<\gamma_{c} \leq 2 T}}\left(\beta_{c}-b\right)+O(1)= & \int_{T}^{2 T} \log |\ell(b+i t, \mathcal{Q})| \mathrm{d} t \\
& -\int_{T}^{2 T} \log |\ell(a+i t, \mathcal{Q})| \mathrm{d} t \\
& -\int_{b}^{a} \arg \ell(\sigma+i T, \mathcal{Q}) \mathrm{d} \sigma \\
& +\int_{b}^{a} \arg \ell(\sigma+2 i T, \mathcal{Q}) \mathrm{d} \sigma \\
= & \sum_{j=1}^{4} I_{j},
\end{aligned}
$$

say. We start with the vertical integrals. Obviously,

$$
I_{1}=\int_{T}^{2 T} \log |\zeta(b+i t ; \mathcal{Q})-c| \mathrm{d} t+T \log \left|\alpha \beta^{b}\right|
$$

Note that

$$
|\zeta(b+i t ; \mathcal{Q})-c|^{2} \leq 2\left(\left.\zeta(b+i t ; \mathcal{Q})\right|^{2}+|c|^{2}\right) .
$$

Thus, we get by applying Jensen's inequality

$$
I_{1} \leq T \log \left(\frac{1}{T} \int_{T}^{2 T}|\zeta(b+i t ; \mathcal{Q})|^{2} \mathrm{~d} t\right)+O(T) .
$$

By Lemma 4 , this is $\ll T$ for $b>\frac{n-1}{2}$. Next we consider $I_{2}$. In view of (22) we find, for sufficiently large $a>\frac{n}{2}$,

(28) $I_{2} \ll \operatorname{Re} \int_{T}^{2 T} \log \left(1+\lambda^{-a-i t}+O\left(\Lambda^{-a}\right) \mathrm{d} t \ll \operatorname{Re} \int_{T}^{2 T} \lambda^{-a-i t} \mathrm{~d} t \ll 1\right.$.

It remains to estimate the horizontal integrals $I_{3}, I_{4}$. Suppose that $\operatorname{Re} \ell(\sigma+i T, \mathcal{Q})$ has $N$ zeros for $b \leq \sigma \leq a$. Then divide $[b, a]$ into at most $N+1$ subintervals in each of which $\operatorname{Re} \ell(\sigma+i T, \mathcal{Q})$ is of constant sign. Then

$$
|\arg \ell(\sigma+i T, \mathcal{Q})| \leq(N+1) \pi
$$

To estimate $N$ let

$$
g(z)=\frac{1}{2}(\ell(z+i T, \mathcal{Q})+\overline{\ell(\bar{z}+i T, \mathcal{Q})}) .
$$


Then we have $g(\sigma)=\operatorname{Re} \ell(\sigma+i T, \mathcal{Q})$. Let $R=a-b$ and choose $T$ so large that $T>2 R$. Now, $\operatorname{Im}(z+i T)>0$ for $|z-a|<T$. Thus $\ell(z+i T, \mathcal{Q})$, and hence $g(z)$ is analytic for $|z-a|<T$. Let $n(r)$ denote the number of zeros of $g(z)$ in $|z-a| \leq r$. Obviously, we have

$$
\int_{0}^{2 R} \frac{n(r)}{r} \mathrm{~d} r \geq n(R) \int_{R}^{2 R} \frac{\mathrm{d} r}{r}=n(R) \log 2 .
$$

With Jensen's formula (see for example [31, §3.61]),

$$
\int_{0}^{2 R} \frac{n(r)}{r} \mathrm{~d} r=\frac{1}{2 \pi} \int_{0}^{2 \pi} \log \left|g\left(a+2 R e^{i \theta}\right)\right| \mathrm{d} \theta-\log |g(a)|,
$$

we deduce

$$
n(R) \leq \frac{1}{2 \pi \log 2} \int_{0}^{2 \pi} \log \left|g\left(a+2 R e^{i \theta}\right)\right| \mathrm{d} \theta-\frac{\log |g(a)|}{\log 2} .
$$

By (22) it follows that $\log |g(a)|$ is bounded. By (19), in any vertical strip of bounded width,

$$
\zeta(s ; \mathcal{Q}) \ll|t|^{B}
$$

with a certain positive constant $B$. Obviously, the same estimate holds for $g(z)$ (at least with a slightly larger constant $B$ ). Thus, the integral above is $\ll \log T$, and $n(R) \ll \log T$. Since the interval $(b, a)$ is contained in the disc $|z-a| \leq R$, the number $N$ is less than or equal to $n(R)$. Therefore, with (29), we get

$$
\left|I_{4}\right| \leq \int_{b}^{a}|\arg \ell(\sigma+i T, \mathcal{Q})| \mathrm{d} \sigma \ll \log T
$$

Obviously, $I_{3}$ can be bounded in the same way. Collecting all estimates, we obtain (13).

Our next aim is to show (14). Let $\sigma>\frac{n-1}{2}$ and fix $b \in\left(\frac{n-1}{2}, \sigma\right)$. Then

$$
\mathcal{N}_{c}^{+}(\sigma, T ; \mathcal{Q}) \leq \frac{1}{\sigma-b} \sum_{\substack{\beta_{c}>\sigma \\ T<\gamma_{c} \leq 2 T}}\left(\beta_{c}-b\right) .
$$

Thus, (13) implies (14).

Next we prove Theorem 1. Here we have to include most of the $c$-values into our observations. First, we note that there exist positive constants $C_{0}, T_{0}$, depending only on $\mathcal{Q}$ and $c$, such that there are no $c$-values of $\zeta(s ; \mathcal{Q})$ in the region $\sigma<-C_{0}, t \geq T_{0}$. In fact, it is a simple consequence of $(16),(19)$, and (22) that

$$
\zeta(s ; \mathcal{Q}) \gg t^{\frac{n}{2}-\sigma}
$$

for sufficiently small $\sigma<0$ and sufficiently large $t$. 
Now assume that $b<-C_{0}-1$ and $t \geq T \geq T_{0}+1$. Then, by (31),

$$
\begin{aligned}
\zeta(b+i t ; \mathcal{Q})-c & =\zeta(b+i t ; \mathcal{Q})\left(1-\frac{c}{\zeta(b+i t ; \mathcal{Q})}\right) \\
& =\zeta(b+i t ; \mathcal{Q})\left(1+O\left(T^{-C_{0}-\frac{n}{2}}\right)\right) .
\end{aligned}
$$

Thus, by (16),

$$
\begin{aligned}
\log |\zeta(b+i t ; \mathcal{Q})-c|= & \log |\Xi(b+i t ; \mathcal{Q})| \\
& +\log \left|\zeta\left(\frac{n}{2}-b-i t ; \mathcal{Q}^{-1}\right)\right|+O\left(T^{-C_{0}-\frac{n}{2}}\right) .
\end{aligned}
$$

In view of $(18)$

$$
\log |\Xi(b+i t, \mathcal{Q})|=-\frac{1}{2} \log (\operatorname{det} \mathcal{Q})+\left(\frac{n}{2}-2 b\right) \log \frac{t}{\pi}+O\left(T^{-1}\right) .
$$

Now (27) gives

$$
\begin{aligned}
I_{1}= & T\left(\log \left|\alpha \beta^{b}\right|-\frac{1}{2} \log (\operatorname{det} \mathcal{Q})\right)+\left(\frac{n}{2}-2 b\right) \int_{T}^{2 T} \log \frac{t}{\pi} \mathrm{d} t \\
& +\int_{T}^{2 T} \log \left|\zeta\left(\frac{n}{2}-b-i t ; \mathcal{Q}^{-1}\right)\right| \mathrm{d} t+O(\log T) .
\end{aligned}
$$

The first integral on the right hand side is easily calculated by elementary means. For the second one note that (23) implies

$$
\zeta\left(s ; \mathcal{Q}^{-1}\right)=\frac{\mathrm{N}\left(\mathcal{Q}^{-1}\right)}{\mathrm{m}_{1}\left(\mathcal{Q}^{-1}\right)^{s}}\left(1+\lambda^{-s}+O\left(\Lambda^{-\sigma}\right)\right)
$$

for some constants $\lambda, \Lambda$ with $\mathrm{m}_{1}\left(\mathcal{Q}^{-1}\right)<\lambda<\Lambda$, depending only on $\mathcal{Q}^{-1}$, as $\sigma \rightarrow \infty$. Hence,

$$
\begin{aligned}
& \int_{T}^{2 T} \log \left|\zeta\left(\frac{n}{2}-b-i t ; \mathcal{Q}\right)\right| \mathrm{d} t \\
& =\int_{T}^{2 T}\left(\log \mathrm{N}\left(\mathcal{Q}^{-1}\right)+\left(b-\frac{n}{2}\right) \log \mathrm{m}_{1}\left(\mathcal{Q}^{-1}\right)+\log \left|1+\left(\lambda^{\frac{n}{2}-b-i t}+o(1)\right)\right|\right) \mathrm{d} t \\
& =T\left(\log \mathrm{N}\left(\mathcal{Q}^{-1}\right)+\left(b-\frac{n}{2}\right) \log \mathrm{m}_{1}\left(\mathcal{Q}^{-1}\right)\right)+O(1)
\end{aligned}
$$

for sufficiently small $b<0$. Thus we get

$$
\begin{aligned}
I_{1}= & \left(\frac{n}{2}-2 b\right) T \log \frac{4 T}{\pi e \sqrt{\mathrm{m}_{1}\left(\mathcal{Q}^{-1}\right)|\beta|}} \\
& +T \log \left(\frac{|\alpha| \mathrm{N}\left(\mathcal{Q}^{-1}\right)}{\sqrt{\operatorname{det} \mathcal{Q}}}\left(\frac{|\beta|}{\mathrm{m}_{1}\left(\mathcal{Q}^{-1}\right)}\right)^{\frac{n}{4}}\right)+O(\log T) .
\end{aligned}
$$


By (26) and with the foregoing estimates for the $I_{j}$ 's we obtain

$$
\begin{aligned}
\sum_{T<\gamma_{c} \leq 2 T}\left(\beta_{c}-b\right)= & \left(\frac{n}{4}-b\right) \frac{T}{\pi} \log \frac{4 T}{\pi e \sqrt{\mathrm{m}_{1}\left(\mathcal{Q}^{-1}\right)|\beta|}} \\
& +\frac{T}{\pi} \log \left(\frac{|\alpha| \mathrm{N}\left(\mathcal{Q}^{-1}\right)}{\sqrt{\operatorname{det} \mathcal{Q}}}\left(\frac{|\beta|}{\mathrm{m}_{1}\left(\mathcal{Q}^{-1}\right)}\right)^{\frac{n}{4}}\right)+O(\log T) .
\end{aligned}
$$

We can rewrite the sum over the $c$-values as follows

$$
\sum_{\beta_{c}}\left(\beta_{c}-b\right)=\left(\frac{n}{4}-b\right) \sum_{\beta_{c}} 1+\sum_{\beta_{c}}\left(\beta_{c}-\frac{n}{4}\right) .
$$

The first sum on the right counts the number of $c$-values and the second one measures the distances of the $c$-values from the critical line. Thus, we obtain

$$
\mathcal{N}_{c}(T ; \mathcal{Q})=\frac{T}{\pi} \log \frac{4 T}{\pi e \sqrt{\mathrm{m}_{1}\left(\mathcal{Q}^{-1}\right)|\beta|}}+O(\log T),
$$

and

$$
\sum_{T<\gamma_{c} \leq 2 T}\left(\beta_{c}-\frac{n}{4}\right)=\frac{T}{\pi} \log \left(\frac{|\alpha| \mathrm{N}\left(\mathcal{Q}^{-1}\right)}{\sqrt{\operatorname{det} \mathcal{Q}}}\left(\frac{|\beta|}{\mathrm{m}_{1}\left(\mathcal{Q}^{-1}\right)}\right)^{\frac{n}{4}}\right)+O(\log T),
$$

with $\alpha$ and $\beta$ corresponding to $\ell=\ell_{j}$. To be more specific, we obtain

$$
\begin{aligned}
\mathcal{N}_{c}(T ; \mathcal{Q}) & =\frac{T}{\pi} \log \frac{4 T}{\pi e \sqrt{\mu(c, \mathcal{Q}) \mathrm{m}_{1}\left(\mathcal{Q}^{-1}\right)}}+O(\log T), \\
\sum_{T<\gamma_{c} \leq 2 T}\left(\beta_{c}-\frac{n}{4}\right) & =\frac{T}{\pi} \log \frac{\nu(c, \mathcal{Q}) \mathrm{N}\left(\mathcal{Q}^{-1}\right)}{\sqrt{\operatorname{det} \mathcal{Q}} \mathrm{m}_{1}\left(\mathcal{Q}^{-1}\right)^{\frac{n}{4}}}+O(\log T),
\end{aligned}
$$

where the quantities $\mu(c ; \mathcal{Q})$ and $\nu(c ; \mathcal{Q})$ are defined by $(10)$ and $(11)$, respectively.

The distribution of the $c$-values close to the real axis is quite regularly. It can be shown that there is always a $c$-value in a neighborhood of any trivial zero of $\zeta(s ; \mathcal{Q})$ with sufficiently large negative real part, and with finitely many exceptions there are no other in the left half-plane. The main ingredients for the proof are Rouché's theorem, Stirling's formula (18), and, for the second claim, estimate (31). Consequently, the number of these $c$-values having real part in $[-R, 0)$ is asymptotically $R$. Thus the contribution of these $c$-values is bounded. On the other side, by (22), the behavior nearby the positive real axis is very regularly. 
Using Corollary (10) with $2^{-n} T$ for $n \in \mathbb{N}$ instead of $T$ and adding up, we get, for fixed $\sigma \leq 0$,

$$
\begin{aligned}
N_{c}(\sigma, T ; \mathcal{Q})= & \sum_{n=1}^{\infty} \mathcal{N}_{c}\left(\sigma, 2^{-n} T\right) \\
= & \frac{T}{\pi}\left(\log \frac{T}{\pi e \sqrt{\mu(c, \mathcal{Q}) \mathrm{m}_{1}\left(\mathcal{Q}^{-1}\right)}} \sum_{n=1}^{\infty} \frac{1}{2^{n}}+\sum_{n=1}^{\infty} \frac{\log 4-n \log 2}{2^{n}}\right) \\
& +O(\log T) .
\end{aligned}
$$

The appearing infinite series are easily evaluated by 1 and 0 , respectively. Hence, this summation removes the factor 4 in the logarithmic term, and we obtain (8). Formula (9) follows by the same argument. Theorem 1 is proved.

It remains to show (15). For this aim let $0<\sigma \leq \frac{n-1}{2}+\epsilon$ and $b$ be a parameter. We decompose

$$
\sum_{\substack{\beta_{c} \\ T<\gamma_{c} \leq 2 T}}\left(\beta_{c}-b\right)=\sum_{\substack{\beta_{c} \leq \sigma \\ T<\gamma_{c} \leq 2 T}}\left(\beta_{c}-b\right)+\sum_{\substack{\sigma<\beta_{c} \leq \frac{n-1}{2}+\epsilon \\ T<\gamma_{c} \leq 2 T}}\left(\beta_{c}-b\right)+\sum_{\substack{c>\frac{n-1}{2} \\ T<\gamma_{c} \leq 2 T}}\left(\beta_{c}-b\right) .
$$

The first sum on the right is equal to

$$
\begin{aligned}
\sum_{\substack{\beta_{c} \leq \sigma \\
T<\gamma_{c} \leq 2 T}}\left(\frac{n-1}{2}+\epsilon-b+\beta_{c}-\frac{n-1}{2}-\epsilon\right) \\
=\sum_{\substack{\beta_{c} \leq \sigma \\
T<\gamma_{c} \leq 2 T}}\left(\beta_{c}-\frac{n-1}{2}-\epsilon\right)+\left(\frac{n-1}{2}+\epsilon-b\right) \sum_{\substack{\beta_{c} \leq \sigma \\
T<\gamma_{c} \leq 2 T}} 1 \\
\leq-\left(\frac{n-1}{2}+\epsilon-\sigma\right) \sum_{\substack{\beta_{c} \leq \sigma \\
T<\gamma_{c} \leq 2 T}} 1+\left(\frac{n-1}{2}+\epsilon-b\right) \sum_{\substack{\beta_{c} \leq \sigma \\
T<\gamma_{c} \leq 2 T}} 1
\end{aligned}
$$

since $\beta_{c} \leq \sigma$. For the second sum we have

$$
\sum_{\substack{\sigma<\beta_{c} \leq \frac{n-1}{2}+\epsilon \\ T<\gamma_{c} \leq 2 T}}\left(\beta_{c}-b\right) \leq\left(\frac{n-1}{2}+\epsilon-b\right) \sum_{\substack{\sigma<\beta_{c} \leq \frac{n-1}{2}+\epsilon \\ T<\gamma_{c} \leq 2 T}} 1 .
$$

Finally, we note for the third sum

$$
\sum_{\substack{\beta_{c}>\frac{n-1}{2}+\epsilon \\ T<\gamma_{c} \leq 2 T}}\left(\beta_{c}-b\right)=\sum_{\substack{\beta_{c}>\frac{n-1}{2}+\epsilon \\ T<\gamma_{c} \leq 2 T}}\left(\beta_{c}-\left(\frac{n-1}{2}+\epsilon\right)\right)+\left(\frac{n-1}{2}+\epsilon-b\right) \sum_{\substack{\beta_{c}>\frac{n-1}{2}+\epsilon \\ T<\gamma_{c} \leq 2 T}} 1,
$$


where the first sum on the right is $\ll T$ by (13). Hence,

$$
\begin{gathered}
\left(\frac{n-1}{2}+\epsilon-\sigma\right) \sum_{\substack{\beta_{c} \leq \sigma \\
T<\gamma_{c} \leq 2 T}} 1 \leq\left(\frac{n-1}{2}+\epsilon-b\right) \\
\sum_{\substack{\beta_{c} \leq \sigma \\
T<\gamma_{c} \leq 2 T}} 1+\left(\frac{n-1}{2}+\epsilon-b\right) \sum_{\substack{\sigma<\beta_{c} \leq \frac{n-1}{2}+\epsilon \\
T<\gamma_{c} \leq 2 T}} 1+\left(\frac{n-1}{2}+\epsilon-b\right) \\
\sum_{\substack{\beta_{c}>\frac{n-1}{2}+\epsilon \\
T<\gamma_{c} \leq 2 T}} 1-\sum_{\substack{\beta_{c} \\
T<\gamma_{c} \leq 2 T}}\left(\beta_{c}-b\right)+O(T) \leq\left(\frac{n-1}{2}+\epsilon-b\right) \\
\sum_{\substack{\beta_{c} \\
T<\gamma_{c} \leq 2 T}} 1-\sum_{\substack{\beta_{c} \\
T<\gamma_{c} \leq 2 T}}\left(\beta_{c}-b\right)+O(T) .
\end{gathered}
$$

In view of (32) we deduce, for sufficiently small $b$,

$$
\mathcal{N}_{c}^{-}(\sigma, T ; \mathcal{Q}) \leq \frac{\frac{n}{4}-\frac{1}{2}+\epsilon}{\frac{n-1}{2}+\epsilon-\sigma} \mathcal{N}_{c}(T ; \mathcal{Q})+O(T)
$$

Taking into account (33) we get (15); it should be noted that this estimate is trivial for $\sigma \geq \frac{n}{4}$. This finishes the proof of Theorem 2 .

\section{Nevanlinna theory}

We conclude with an application of Theorem 1 to Nevanlinna theory. This theory was introduced by Rolf Nevanlinna in the 1920's to tackle the value-distribution of meromorphic functions in general. First, we recall some basic facts which, for example, can be found in Nevanlinna's monograph [23, Chapters VI and IX].

Let $f$ be a meromorphic function and denote the number of poles of $f(s)$ in $|s|<r$ by $\mathbf{n}(f, \infty, r)$ (counting multiplicities). The number of $c$-values of $f$ is given by

$$
\mathbf{n}(f, c, r)=\mathbf{n}\left(\frac{1}{f-c}, \infty, r\right) .
$$

Then the integrated counting function is given by

$$
\mathbf{N}(f, c, r)=\int_{0}^{r}(\mathbf{n}(f, c, \rho)-\mathbf{n}(f, c, 0)) \frac{\mathrm{d} \rho}{\rho}+\mathbf{n}(f, c, 0) \log r .
$$


The proximity function is defined by

$$
\mathbf{m}(f, r)=\frac{1}{2 \pi} \int_{0}^{2 \pi} \log ^{+}|f(r \exp (i \theta))| \mathrm{d} \theta
$$

and

$$
\mathbf{m}(f, c, r)=\mathbf{m}\left(\frac{1}{f-c}, r\right)
$$

where $\log ^{+} x:=\max (0, \log x)$. The function $\mathbf{m}(f, c, r)$ indicates how close $f(s)$ is to the $c$-values on the circle $|s|=r$. The characteristic function of $f$ is defined by

$$
\mathbf{T}(f, r)=\mathbf{N}(f, \infty, r)+\mathbf{m}(f, r) .
$$

Furthermore, let

$$
\mathbf{T}(f, c, r)=\mathbf{N}(f, c, r)+\mathbf{m}(f, c, r) .
$$

The first main theorem in Nevanlinna theory states that $\mathbf{T}(f, c, r)$ differs from the characteristic function by a bounded quantity:

Lemma 6. Let $f$ be a meromorphic function and let $c$ be any complex number. Then

$$
\mathbf{T}(f, c, r)=\mathbf{T}(f, r)+O(1) .
$$

The characteristic function $\mathbf{T}(f, r)$ encodes interesting information about the analytic behaviour of $f$. The quantity

$$
\delta(f, c):=1-\limsup _{r \rightarrow \infty} \frac{\mathbf{N}(f, c, r)}{\mathbf{T}(f, r)}
$$

is called the deficiency of the value $c$ of $f$. This deficiency is positive only if there are relatively few $c$-values.

Only recently Ye $[\mathbf{3 3}]$ computed the Nevanlinna functions for the Riemann zeta-function. This was extended by the author $[\mathbf{2 8}],[\mathbf{2 9}]$ to functions in the Selberg class. As an application of our previous results we shall extend these results to Epstein zeta-functions.

Firstly, let $\sigma_{0}>\frac{n}{2}$ be fixed. We write $s=r \exp (i \theta)$, so $\sigma=r \cos \theta$. It is easily seen that, for $\sigma>\sigma_{0}$,

$$
\frac{1}{2 \pi} \int_{\left\{\theta \in[0,2 \pi]: r \cos \theta>\sigma_{0}\right\}} \log ^{+}|\zeta(r \exp (i \theta) ; \mathcal{Q})| \mathrm{d} \theta \ll 1 .
$$

Further, in view of Theorem 1,

$$
\frac{1}{2 \pi} \int_{\left\{\theta \in[0,2 \pi] \mid \frac{n}{2}-\sigma_{0} \leq r \cos \theta \leq \sigma_{0}\right\}} \log ^{+}|\zeta(r \exp (i \theta) ; \mathcal{Q})| \mathrm{d} \theta \ll \log r
$$


for $\frac{n}{2}-\sigma_{0} \leq \sigma \leq \sigma_{0}$; note that the Lebesgue measure of the set

$$
\left\{\theta \in[0,2 \pi] \mid \sigma=r \cos \theta \in\left[\frac{n}{2}-\sigma_{0}, \sigma\right]\right\}
$$

is bounded by $O\left(\frac{1}{r}\right)$. Finally, we have for $\sigma \leq \frac{n}{2}-\sigma_{0}$ by the functional equation in the form (16)

$$
\begin{aligned}
\log ^{+}|\zeta(r \exp (i \theta) ; \mathcal{Q})| \leq & \log ^{+}\left|\Gamma\left(\frac{n}{2}-r \exp (i \theta)\right)\right| \\
& +\log ^{+}|\Gamma(r \exp (i \theta))|+O(r) .
\end{aligned}
$$

Now we shall use Ye's decomposition of the Gamma-function. For any $z=r \exp (i \theta)$, there is an integer $n_{0}$ with $n_{0}<r \leq n_{0}+1$ such that

$$
\frac{1}{\Gamma(z)}=F_{1}(z) F_{2}(z) \quad \text { with } \quad F_{1}(z):=z\left(\gamma z-\sum_{n=1}^{2 n_{0}} \frac{z}{n}\right)
$$

where $\gamma$ is the Euler-Mascheroni constant, and $F_{2}(z)$ is an entire function with $\mathbf{m}\left(F_{2}, r\right) \ll r$. The order of growth of $\Gamma(z)$ is ruled by the order of growth of $F_{1}(z)$. Ye computed

$$
\log \left|F_{1}(z)\right|=-r \log r \cos \theta+O(r) \text {. }
$$

This leads to

$$
\begin{aligned}
&\left.\frac{1}{2 \pi} \int_{\{\theta \in[0,2 \pi] \mid r \cos \theta}<1-\sigma_{0}\right\} \log ^{+}\left|\Gamma\left(\frac{n}{2}-r \exp (i \theta)\right)\right| \mathrm{d} \theta \\
& \leq \frac{1}{2 \pi} \int_{-\frac{\pi}{2}}^{\frac{\pi}{2}} r \log r \cos \theta \mathrm{d} \theta+O(r)=\frac{r}{\pi} \log r+O(r)
\end{aligned}
$$

and, similarly,

$$
\frac{1}{2 \pi} \int_{\left\{\theta \in[0,2 \pi] \mid r \cos \theta<\frac{n}{2}-\sigma_{0}\right\}} \log ^{+}|\Gamma(r \exp (i \theta))| \mathrm{d} \theta \leq \frac{r}{\pi} \log r+O(r) .
$$

Thus we get

$$
\frac{1}{2 \pi} \int_{\left\{\theta \in[0,2 \pi] \mid r \cos \theta<\frac{n}{2}-\sigma_{0}\right\}} \log ^{+}|\zeta(r \exp (i \theta) ; \mathcal{Q})| \mathrm{d} \theta \leq \frac{r}{\pi} \log r+O(r) .
$$

Adding the estimates for the other cases we obtain for the proximity function of $\zeta(s ; \mathcal{Q})$, which we shall abbreviate by $\zeta$ in the sequel, now easily

$$
\mathbf{m}(\zeta, r) \leq \frac{2 r}{\pi} \log r+O(r)
$$

Since $\zeta(s ; \mathcal{Q})$ is regular except for at most a pole at $s=\frac{n}{2}$,

$$
\mathbf{N}(\zeta, \infty, r) \ll \int_{1}^{r} \frac{\mathrm{d} \rho}{\rho}=\log r .
$$


Thus we get

$$
\mathbf{T}(\zeta, r) \leq \frac{2 r}{\pi} \log r+O(r)
$$

It follows from Theorem 1, Equation (12), and the fact that the contribution of the trivial zeros of modulus less than $r$ is $O(r)$, that

$$
\mathbf{N}(\zeta, 0, r)=\frac{2 r}{\pi} \log r+O(r) .
$$

The first main theorem, Lemma 6, implies

$$
\begin{aligned}
\mathbf{N}(\zeta, 0, r) \leq \mathbf{T}(\zeta, 0, r) & =\mathbf{T}(\zeta, r)+O(1) \\
& =\mathbf{N}(\zeta, \infty, r)+\mathbf{m}(\zeta, r)+O(r) .
\end{aligned}
$$

In view of (35) and (36) we get an asymptotic formula for the characteristic function:

Theorem 7. As $r \rightarrow \infty$,

$$
\mathbf{T}(\zeta, r)=\frac{2 r}{\pi} \log r+O(r) .
$$

We deduce from this and (34) for the deficiency value of infinity

$$
\delta(\zeta, \infty)=1-\limsup _{r \rightarrow \infty} \frac{\mathbf{N}(\zeta, \infty, r)}{\mathbf{T}(\zeta, r)}=1,
$$

as expected. In view of Theorem 1 the deficiency values for $c \neq \infty$ are equal to 0 .

We conclude with a description of the analytic behavior of Epstein zeta-functions in terms of the notion of finite order. A positive function $t(r)$ is said to be of finite order $\lambda$ if

$$
\limsup _{r \rightarrow \infty} \frac{\log t(r)}{\log r}=\lambda
$$

$t(r)$ is of maximum, mean or minimum type of order $\lambda$ if the upper limit

$$
\limsup _{r \rightarrow \infty} \frac{t(r)}{r^{\lambda}}
$$

is infinite, finite and positive, or zero. A meromorphic function is defined to be of the same order and the same type as its characteristic function $\mathbf{T}(r, f)$. Thus, by Theorem 7 , we get

Corollary 8. Every Epstein zeta-function is of order one and maximum type. 
Acknowledgements. The author wants to thank the organizers of the conference 'Primeras Jornadas de Teoría de Números' held in Vilanova i la Geltrú (Barcelona), June 30-July 2, 2005 for their kind hospitality. Moreover, he is grateful to the Spanish Ministry for Science and Education for having the opportunity to work from December 2004 to March 2006 as 'Rámon y Cajal Investigador' at the Departamento de Matemáticas, Universidad Autónoma de Madrid, and he also wishes to thank his colleagues for this wonderful time.

\section{References}

[1] P. T. Bateman and E. Grosswald, On Epstein's zeta function, Acta Arith. 9 (1964), 365-373.

[2] E. Bombieri And D. A. Hejhal, On the zeros of Epstein zeta functions, C. R. Acad. Sci. Paris Sér. I Math. 304(9) (1987), 213-217.

[3] R. E. Crandall and J. P. Buhler, Elementary function expansions for Madelung constants, J. Phys. A 20(16) (1987), 5497-5510.

[4] K. Chandrasekharan and R. Narasimhan, The approximate functional equation for a class of zeta-functions, Math. Ann. 152 (1963), 30-64.

[5] S. Chowla and A. Selberg, On Epstein's zeta function. I, Proc. Nat. Acad. Sci. U. S. A. 35 (1949), 371-374.

[6] J. H. Conway and N. J. A. Sloane, "Sphere packings, lattices and groups", Second edition, With additional contributions by E. Bannai, R. E. Borcherds, J. Leech, S. P. Norton, A. M. Odlyzko, R. A. Parker, L. Queen and B. B. Venkov, Grundlehren der Mathematischen Wissenschaften 290, Springer-Verlag, New York, 1993.

[7] H. Davenport and H. Heilbronn, On the zeros of certain Dirichlet series, I, II, J. London Math. Soc. 11 (1936), 181-185, 307-313.

[8] M. Deuring, Zetafunktionen quadratischer Formen, J. reine angew. Math. 172 (1935), 226-252.

[9] W. Duke And R. Schulze-Pillot, Representation of integers by positive ternary quadratic forms and equidistribution of lattice points on ellipsoids, Invent. Math. 99(1) (1990), 49-57.

[10] H. M. EDWARDs, "Riemann's zeta function", Pure and Applied Mathematics 58, Academic Press [A subsidiary of Harcourt Brace Jovanovich, Publishers], New York-London, 1974.

[11] E. Elizalde And A. Romeo, Regularization of general multidimensional Epstein zeta-functions, Rev. Math. Phys. 1(1) (1989), $113-128$. 
[12] P. Epstein, Zur Theorie allgemeiner Zetafunctionen, Math. Ann. 56(4) (1903), 615-644.

[13] O. M. Fomenko, On Epstein's zeta-function, (Russian), Zap. Nauchn. Sem. S.-Peterburg. Otdel. Mat. Inst. Steklov. (POMI) 286 (2002), Anal. Teor. Chisel i Teor. Funkts. 18, 169-178, 231; translation in J. Math. Sci. (N. Y.) 122(6) (2004), 3679-3684.

[14] A. FusiI, On the zeros of the Epstein zeta functions, J. Math. Kyoto Univ. 36(4) (1996), 697-770.

[15] D. A. HejhaL, Zeros of Epstein zeta functions and supercomputers, in: "Proceedings of the International Congress of Mathematicians", Vol. 1, 2 (Berkeley, Calif., 1986), Amer. Math. Soc., Providence, RI, 1987, pp. 1362-1384.

[16] H. IWANIEC, "Topics in classical automorphic forms" Graduate Studies in Mathematics 17, American Mathematical Society, Providence, RI, 1997.

[17] S. Kanemitsu, A. Sankaranarayanan and Y. Tanigawa, A mean value theorem for Dirichlet series and a general divisor problem, Monatsh. Math. 136(1) (2002), 17-34.

[18] A. A. Karatsuba and S. M. Voronin, "The Riemann zetafunction", Translated from the Russian by Neal Koblitz, de Gruyter Expositions in Mathematics 5, Walter de Gruyter \& Co., Berlin, 1992.

[19] M. Koecher and A. Krieg, "Elliptische Funktionen und Modulformen", Springer-Verlag, Berlin, 1998.

[20] E. Landau, Beiträge zur analytischen Zahlentheorie, Rend. Circ. Mat. Palermo 26 (1908), 169-302.

[21] N. Levinson, Almost all roots of $\zeta(s)=a$ are arbitrarily close to $\sigma=1 / 2$, Proc. Nat. Acad. Sci. U.S.A. 72 (1975), 1322-1324.

[22] J. E. Littlewood, On the zeros of the Riemann zeta-function, Proc. Cambridge Phil. Soc. 22 (1924), 295-318.

[23] R. Nevanlinna, "Analytic functions", Translated from the second German edition by Phillip Emig, Die Grundlehren der mathematischen Wissenschaften 162, Springer-Verlag, New York-Berlin, 1970.

[24] H. S. A. Potter and E. C. Titchmarsh, The zeros of Epstein's zeta-functions, Proc. London Math. Soc. 39 (1935), 372-384.

[25] C. L. Siegel, Contributions to the theory of the Dirichlet $L$-series and the Epstein zeta-functions, Ann. of Math. (2) 44 (1943), $143-172$.

[26] C. L. Siegel, "Advanced analytic number theory", Second edition, Tata Institute of Fundamental Research Studies in Mathematics 9, Tata Institute of Fundamental Research, Bombay, 1980. 
[27] H. M. Stark, On the zeros of Epstein's zeta function, Mathematika 14 (1967), 47-55.

[28] J. Steuding, Value-distribution of $L$-functions, Habilitation thesis, Frankfurt (2003), to appear in Lecture Notes of Mathematics, Springer.

[29] J. Steuding, On the value-distribution of L-functions, Fiz. Mat. Fak. Moksl. Semin. Darb. 6 (2003), 87-119.

[30] J. Steuding, On the zero-distribution of Epstein zeta-functions, Math. Ann. 333(3) (2005), 689-697.

[31] E. C. Titchmarsh, "The theory of the Riemann zeta-function", Second edition, Edited and with a preface by D. R. Heath-Brown, The Clarendon Press, Oxford University Press, New York, 1986.

[32] S. M. Voronin, The zeros of zeta-functions of quadratic forms, (Russian), Number theory, mathematical analysis and their applications, Trudy Mat. Inst. Steklov. 142 (1976), 135-147, 269.

[33] Z. YE, The Nevanlinna functions of the Riemann Zeta-function, J. Math. Anal. Appl. 233(1) (1999), 425-435.

Institut für Mathematik

Universität Würzburg

Am Hubland

97074 Würzburg

Germany

E-mail address: steuding@mathematik.uni-wuerzburg.de 